\title{
Formation des collections archéologiques et ethnographiques : notes sur des trajectoires d'objets latino-américains
}

The formation of archaeological and ethnographic collections: notes on the trajectories of Latin American objects

\section{Vanessa Bernal Multon}

\section{(2) OpenEdition}

\section{Journals}

Édition électronique

URL : http://journals.openedition.org/cel/878

DOI : 10.4000/cel.878

ISSN : 2262-208X

Éditeur

École du Louvre

Référence électronique

Vanessa Bernal Multon, « Formation des collections archéologiques et ethnographiques : notes sur des trajectoires d'objets latino-américains », Les Cahiers de l'École du Louvre [En ligne], 12 | 2018, mis en ligne le 03 mai 2018, consulté le 17 septembre 2019. URL : http://journals.openedition.org/cel/878 ; DOI : 10.4000/cel.878

Ce document a été généré automatiquement le 17 septembre 2019.

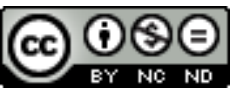

Les Cahiers de l'École du Louvre sont mis à disposition selon les termes de la licence Creative Commons Attribution - Pas d'Utilisation Commerciale - Pas de Modification 4.0 International. 


\title{
Formation des collections
}

\section{archéologiques et ethnographiques : notes sur des trajectoires d'objets latino-américains}

\author{
The formation of archaeological and ethnographic collections: notes on the \\ trajectories of Latin American objects
}

Vanessa Bernal Multon

1 En admirant les vitrines des expositions présentant des objets de cultures ancestrales comme celles des Incas ou des Mayas, les spectateurs sont souvent amenés à se poser de nombreuses questions sur l'histoire de ces peuples, sur leurs croyances, leurs mœurs et leurs traditions. Ces interrogations sont extrêmement intéressantes, mais en y réfléchissant bien, n'existerait-il pas une question fondamentale et tout aussi passionnante à côté de laquelle ces visiteurs passeraient systématiquement ? Ces objets qu'ils peuvent admirer, ces pièces qu'ils ont sous les yeux, véritables témoins chargés d'Histoire et d'histoires, comment sont-ils arrivés dans ces vitrines? Quels sont leurs parcours, les chemins qu'ils ont suivis depuis leur fabrication dans ces contrées lointaines jusqu'à leur arrivée dans l'exposition où tant de gens auront le loisir de les contempler?

Provenant de mondes lointains, très divers et très différents du monde occidental, des milliers d'objets sont arrivés en France tout au long des cinq derniers siècles pour accroître les collections de nombreux musées. Dans quelles conditions ces objets ont-ils entrepris de si longs voyages pour être accueillis ici? Qui sont les aventuriers, les explorateurs, les scientifiques, les collectionneurs qui ont œuvré à leur déplacement, et quels étaient leurs objectifs avoués ou inavoués?

Des questions complémentaires peuvent venir s'ajouter à ces premières interrogations. Comment ces objets lointains ont-ils subitement été considérés comme les témoins fondamentaux de cultures exotiques et sauvages? Comment ces pièces sont-elles devenues le sujet central de nouvelles disciplines scientifiques apparues au début du 
$\mathrm{XIX}^{\mathrm{e}}$ siècle ? Comment le monde occidental, à partir de ces études, a-t-il cru pouvoir véritablement comprendre ces cultures lointaines et s'est peu à peu arrogé le droit de discourir à leur sujet, et d'imposer sa vision et son interprétation de leur histoire? Comment ces objets ont-ils été utilisés dans les discours muséographiques à des fins politiques et coloniales? Et pour finir, comment ces objets se sont-ils fait une place durable au sein du patrimoine français?

2 Ainsi, de nombreuses questions se posent et s'imposent en regardant les collections d'objets provenant d'Afrique, d'Asie, d'Océanie et des Amériques, ces quatre continents qui ont été et qui continuent d'être ces mondes lointains et mystérieux pour l'Europe. Ces interrogations déclenchent une immense curiosité et il est nécessaire d'apporter des réponses à ces énigmes passionnantes, de dévoiler les secrets qui se cachent derrière ces pièces. C'est dans ce contexte que nous avons décidé d'entreprendre une recherche doctorale mais, au vu de la richesse de ces objets dans les collections françaises, en nous concentrant uniquement sur l'Amérique latine. Plus précisément, ce travail de recherche étudiera les faits et les circonstances de l'arrivée des pièces latinoaméricaines en $\mathrm{France}^{1}$, et traitera de leur rassemblement dans les musées afin d'établir une historiographie des collections et de leur présence dans les discours muséographiques. Cette première phase doit ensuite permettre d'établir un lien entre la formation des collections archéologiques et ethnographiques provenant d'Amérique latine au sein des musées français et l'évolution de la vision occidentale de l'Autre.

Cet article se concentrera sur la première partie de ce sujet, à savoir l'histoire de la formation des collections archéologiques et ethnographiques latino-américaines, ainsi que l'histoire des acteurs et des institutions qui sont liés à ces collections et qui ont au fil du temps donné un statut différent aux objets. Pour ce faire, nous prendrons l'exemple de cinq objets soigneusement sélectionnés. Leur arrivée en France est liée à quatre personnages, représentant chacun une époque particulière qui explique le parcours de ces objets.

Dans un premier temps, cet article présentera chacun des objets choisis, avec ses caractéristiques et une description détaillée. Ensuite, l'histoire des personnages qui les ont découverts et apportés en France permettra d'évoquer les conditions de leur arrivée. Finalement, l'analyse de leur «vie » en France, de leur changement de statut produit par des idées ou des contextes politico-historiques particuliers, et symbolisé par l'évolution de leur présentation au public permettra d'amorcer une réflexion sur l'évolution du regard occidental sur l'Autre, sujet central de notre recherche doctorale.

Cinq objets ont donc été sélectionnés pour servir de base à cet article. Si tous ces objets proviennent de cultures préhispaniques du Pérou et du Mexique, ils sont arrivés en France à des époques différentes, par des moyens différents, pour des raisons différentes et grâce à l'intervention de personnalités différentes. L'histoire de ces objets et les trajectoires qu'ils ont prises constituent de parfaits exemples de la richesse et de la diversité des parcours des objets archéologiques ou ethnographiques ${ }^{2}$. Une «vie » qui commence au moment de leur fabrication, qui se poursuit pendant la durée de leur utilisation, qui continue et se renouvelle quand ces objets sont (re)découverts et choisis pour faire partie d'une collection, leur permettant d'acquérir un nouveau statut, qui peut changer au fil du temps en fonction de circonstances très variées (historiques, institutionnelles, politiques, etc.). C'est ainsi de véritables biographies qu'il faudrait écrire sur ces objets, biographies qui « peuvent rendre saillant, ce qui resterait obscur autrement ${ }^{3} »$. 
Le premier objet étudié dans cet article est une chemise (généralement désignée sous son nom quechua uncu) provenant de la côte centrale du Pérou, faite en coton avec une décoration brodée en fibre de camélidé (fig. 1). La décoration principale représente quatre personnages stylisés de face (deux à l'avant de la chemise et deux à l'arrière), levant les bras, qui portent une tunique et une coiffe ${ }^{4}$. En complément de ces figures, des bandeaux de différentes couleurs sont brodés au niveau des poignets, du buste et sur la bordure inférieure de la chemise. Cet objet est actuellement conservé dans les réserves du musée du quai Branly- Jacques Chirac sous le numéro d'inventaire 71.1878.5.39.

Fig. 1

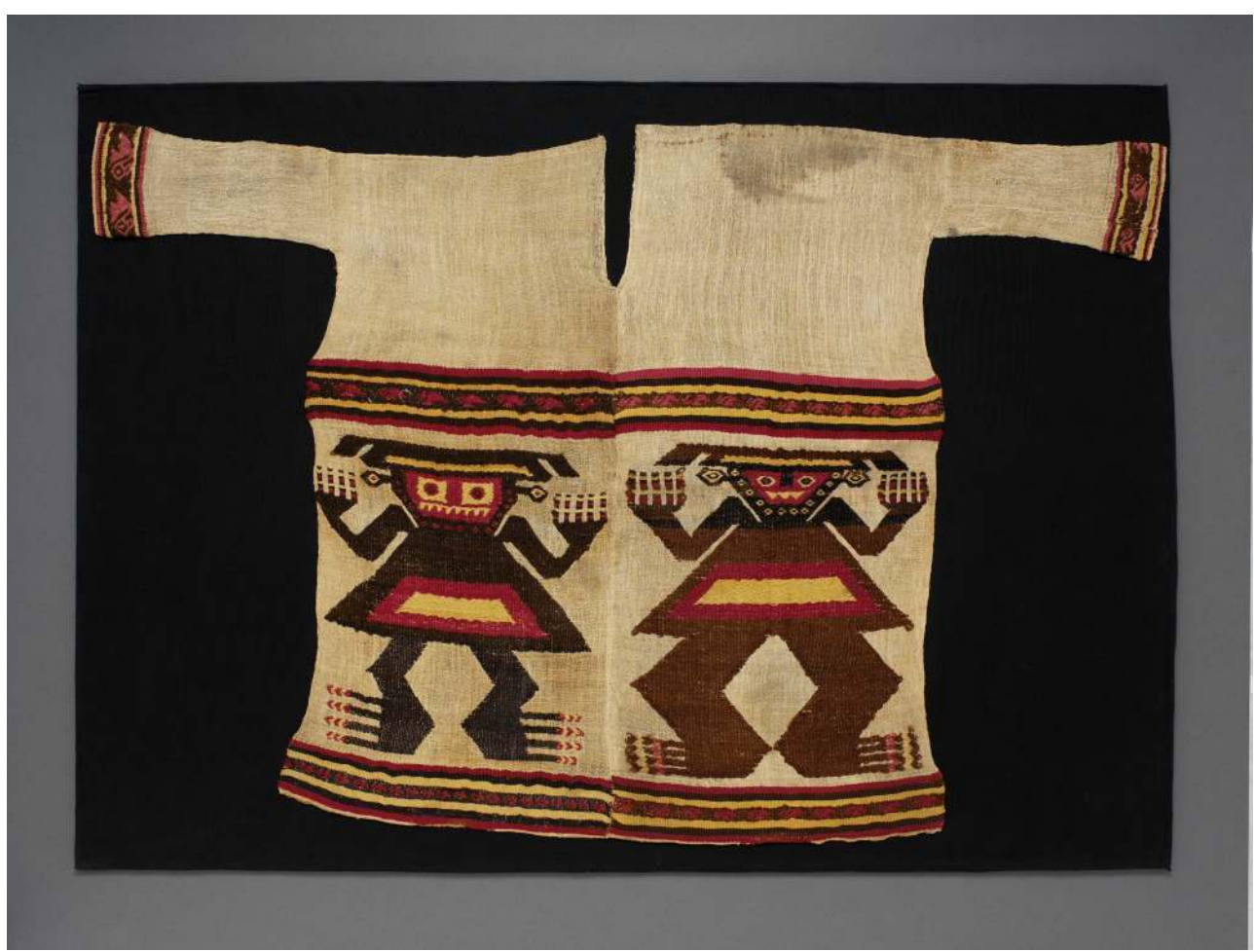

Chemise provenant de la côte centrale du Pérou, dépôt de la Bibliothèque nationale de France, musée du quai Branly - Jacques Chirac, inv. 71.1878.5.39

(c) musée du quai Branly - Jacques Chirac, photo Patrick Gries, Valérie Torre

Le deuxième objet est un vase anthropomorphe de culture inca (fig. 2). Il s'agit d'un personnage vêtu d'une chemise peinte, portant un sac en bandoulière. Il tient dans sa main droite une hache, fixée dans un manche en bois par une épaisse courroie ${ }^{5}$. Il est lui aussi conservé au musée du quai Branly- Jacques Chirac sous le numéro d'inventaire 71.1878.5.30, et il est actuellement exposé sur le plateau des collections permanentes du musée. Ces deux pièces ont été rapportées du Pérou par Joseph Dombey entre 1779 et $1784^{6}$. 
Fig. 2

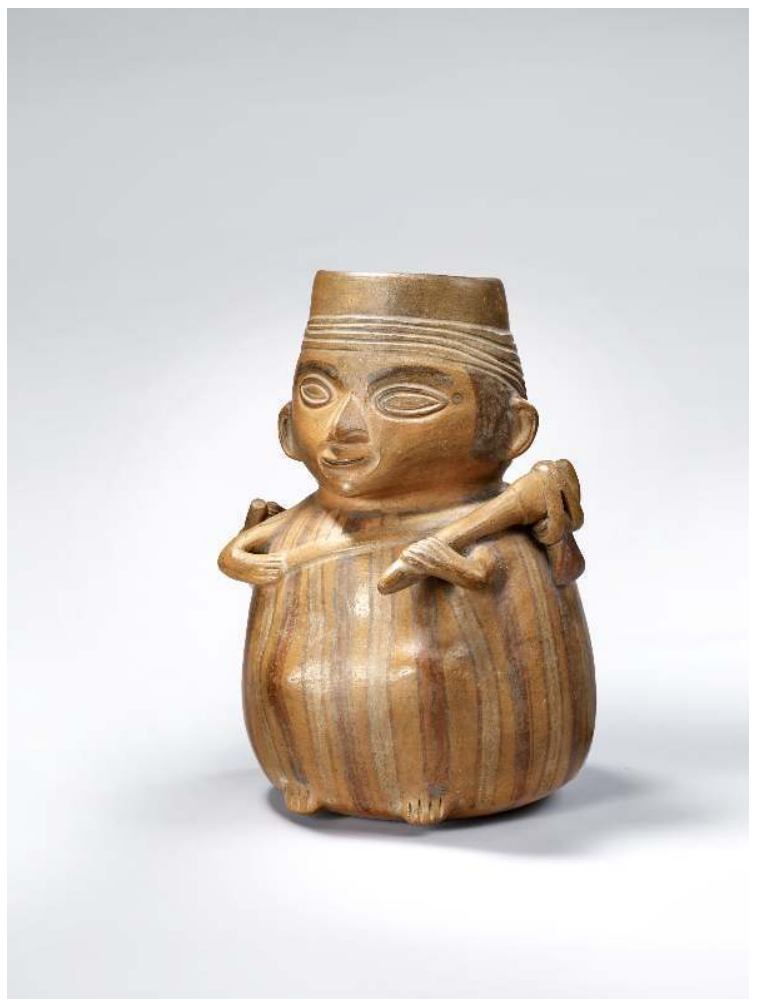

Vase anthropomorphe de la culture inca, dépôt de la Bibliothèque nationale de France, musée du quai Branly - Jacques Chirac, inv. 71.1878.5.30

(C) musée du quai Branly - Jacques Chirac, photo Claude Germain

La troisième pièce est une bouteille phytomorphe à anses, avec un décor géométrique sur les flancs, provenant de la côte centrale du Pérou (fig. 3). Cette pièce a été rapportée en France par le botaniste Alcide d'Orbigny et vendue au musée national de Sèvres en 1834. Elle se trouve actuellement dans les réserves de ce musée sous le numéro d'inventaire MNC 1765. 
Fig. 3

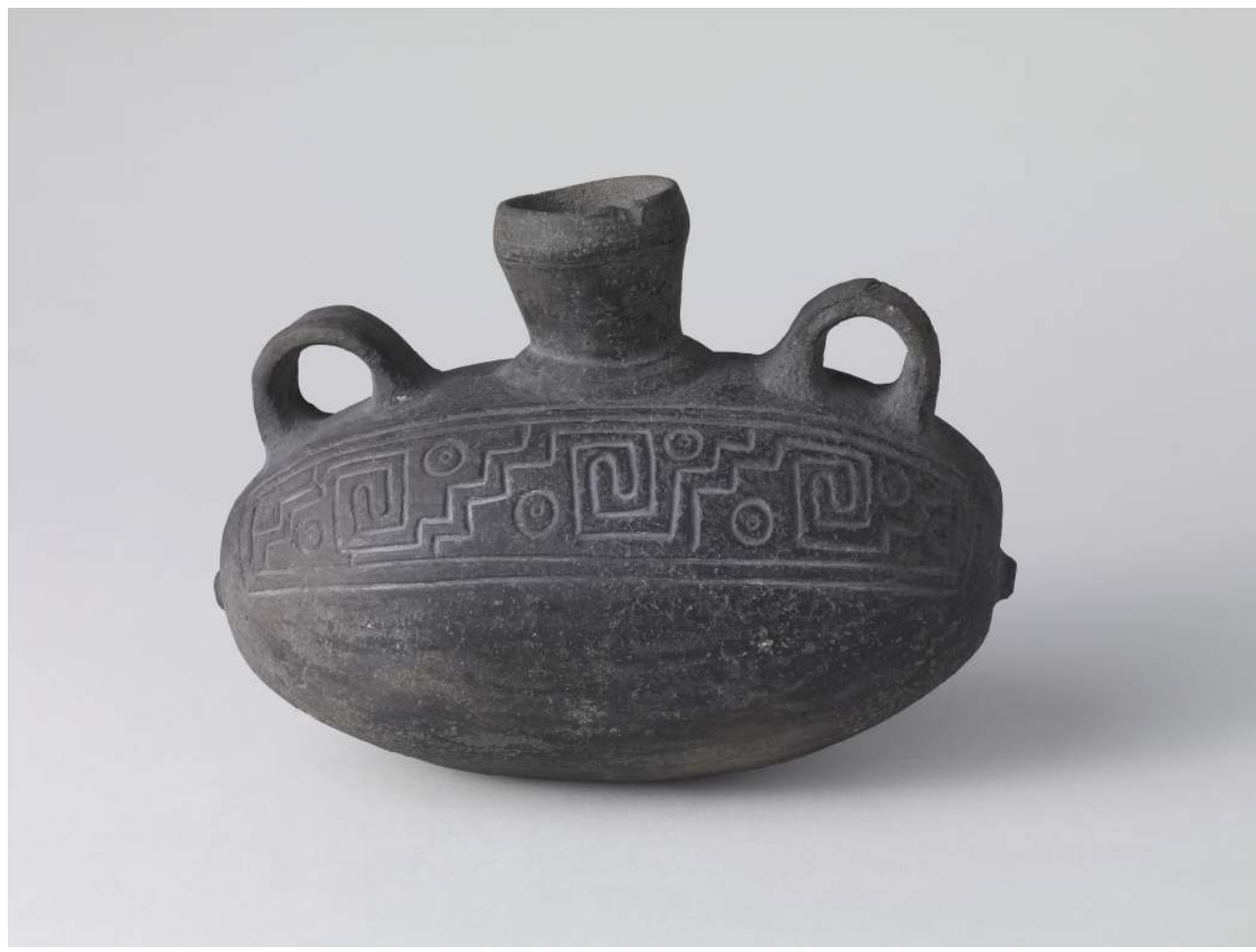

Bouteille phytomorphe à anses provenant de la côte centrale du Pérou, collection Alcide d'Orbigny, musée national de Sèvres, inv. MNC 1765

Photo (C) RMN-Grand Palais (Sèvres, Cité de la céramique) / Martine Beck-Coppola

La quatrième pièce est un masque anthropomorphe en pierre dure sculptée provenant du Mexique (fig.4). Elle fait partie des collections rassemblées par l'explorateur Auguste Génin en 1893, lors de ses explorations archéologiques dans l'État mexicain d'Hidalgo. Actuellement, cette pièce est conservée dans les réserves du musée du quai Branly - Jacques Chirac sous le numéro 71.1897.53.162. 
Fig. 4

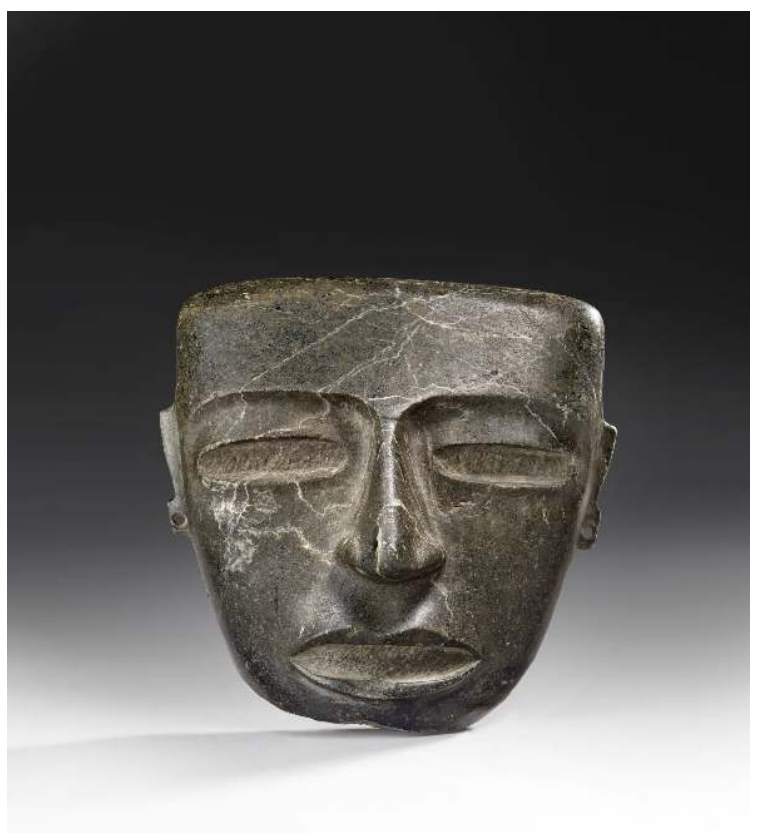

Masque anthropomorphe en pierre provenant du Mexique, collection Auguste Génin, musée du quai Branly - Jacques Chirac, inv. 71.1897.53.162

(c) musée du quai Branly - Jacques Chirac, photo Claude Germain

Enfin, la cinquième et dernière pièce est un masque funéraire Nasca en or, entouré de seize serpents découpés dont deux au sommet se prolongent au-delà de la tête et se terminent par un oiseau (fig. 5). Le décor repoussé comprend, sur le masque, deux yeux, un nez, deux lignes de dents superposées, deux lignes sinueuses descendant de chaque œil sur les joues, des lignes sinueuses descendant de la bouche sur le menton ; sur les serpents, deux yeux et une ligne d'épine dorsale ; sur les oiseaux, un œil, des lignes figurant les plumes des ailes et une ligne courbe sous les pattes. Cette pièce a été vendue par le collectionneur péruvien Guillermo Schmidt-Pizarro en 1930 au musée de l'Homme. Actuellement, ce masque en or est conservé au musée du quai BranlyJacques Chirac, et son numéro d'inventaire est 71.1930.49.1. 
Fig. 5

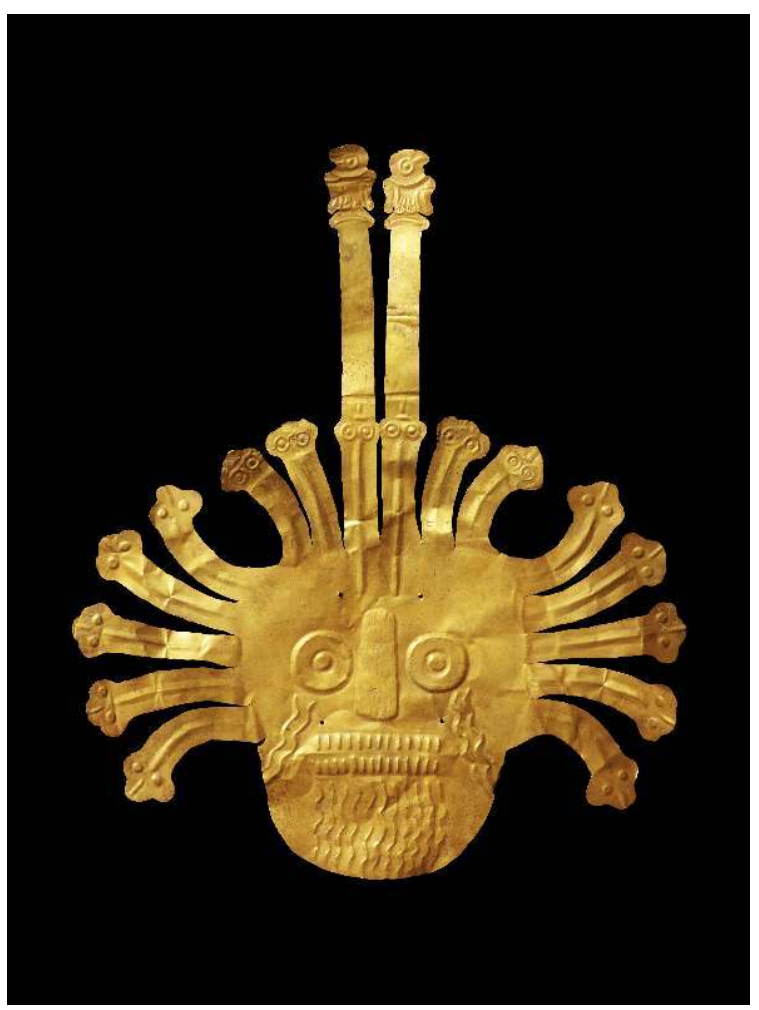

Masque funéraire en or de la culture Nasca, collection Georges Henri Rivière, musée du quai Branly Jacques Chirac, inv. 71.1930.49.1

(c) musée du quai Branly - Jacques Chirac, photo Patrick Gries, Valérie Torre

5 Après cette brève description des cinq pièces dont il sera question dans cette étude, il faut maintenant s'interroger sur la façon dont ces objets sont arrivés en France. Cette question, centrale dans cet article, ne peut être abordée qu'en s'intéressant à l'histoire des quatre personnages dont le parcours et l'action sont à l'origine du destin français des cinq pièces. Chacun de ces personnages a apporté des éléments importants à l'histoire de la formation des collections latino-américaines en Europe. Certains sont très connus, d'autres très peu ; ils appartiennent à quatre époques successives, de la fin $\mathrm{du}$ XVIII ${ }^{\mathrm{e}}$ siècle au Xx $\mathrm{x}^{\mathrm{e}}$ siècle. Ces personnages sont Joseph Dombey, Alcide d'Orbigny, Auguste Génin et Guillermo Schmidt-Pizarro. Tous les quatre ont œuvré à la création d'importantes collections ainsi qu'au passage des pièces d'un continent à un autre, qui marque pour ces objets le début d'une nouvelle vie. Par cette renaissance, les objets acquièrent un nouveau statut, et c'est ce passage particulier que nous allons maintenant aborder.

$6 \mathrm{Au}$ cours du $\mathrm{XVIII}^{\mathrm{e}}$ siècle et au début $\mathrm{du}$ XIX ${ }^{\mathrm{e}}$ siècle, une multitude de missions d'exploration sont envoyées parcourir le monde entier, une part importante de ces missions étant motivée par l'étude de diverses disciplines scientifiques, comme l'histoire naturelle. Il existe en effet une grande volonté d'apprendre, de découvrir, de connaître et de classifier le monde et ces missions ont pour objectif d'acquérir de meilleures connaissances en botanique, en minéralogie, en zoologie, en anatomie, etc. Par exemple, en Amérique du Sud sont organisées les expéditions du botaniste Joseph de Jussieu, du naturaliste et géographe allemand Alexander von Humboldt, du botaniste français Aimé Bonpland, etc. 
C'est dans ce contexte que les naturalistes Joseph Dombey et Alcide d'Orbigny sont envoyés en Amérique du Sud pour réaliser une expédition scientifique, respectivement $\mathrm{au} \mathrm{XVIII}^{\mathrm{e}}$ et au $\mathrm{XIX}^{\mathrm{e}}$ siècle. Leur but principal consiste à rassembler des collections botaniques, mais, pour profiter au maximum de leur séjour en terres exotiques, ils réalisent également de manière complémentaire quelques recherches archéologiques et ethnographiques. Dans l'esprit encyclopédique développé pendant le Siècle des lumières, les deux naturalistes ne doivent pas laisser passer l'opportunité de réaliser tout type de recherche et de rassembler de grandes collections, qui doivent comporter la plus grande variété d'objets provenant de cultures et de sociétés lointaines.

7 La mission de Joseph Dombey marque un changement important dans l'histoire de la formation des collections qui nous intéressent, car c'est une des premières fois que des institutions européennes organisent une mission dans le but de rapporter des objets particuliers avec un intérêt scientifique. Jusqu'alors, ce type d'objets arrive surtout en Europe comme témoin des conquêtes effectuées, à des fins politiques. Ainsi, la collection Dombey est une des premières collections rassemblées à partir de fouilles archéologiques ${ }^{7}$, et les objets qui la composent figurent parmi les premiers arrivés en France dont le parcours peut être retracé d'une manière relativement précise.

Joseph Dombey, naturaliste-explorateur français, est choisi pour faire partie de l'expédition franco-espagnole dirigée par les Espagnols Hipólito Ruiz López et José Pavón, avec comme objectif de mener à bien différentes études en Amérique du Sud. Selon Ernest-Théodore Hamy, directeur du musée d'ethnographie du Trocadéro, ce projet d'expédition est suscité par Anne-Robert-Jacques Turgot $^{8}$. Cette idée est confirmée par Catherine Lang qui affirme que c'est le "Contrôleur général des Finances, Anne-Robert-Jacques Turgot qui projetait une importante mission scientifique afin d'étudier et de naturaliser des végétaux ${ }^{9}$ ». Ainsi, la couronne française présente à la couronne espagnole le projet de cette expédition dans la vice-royauté du Pérou, et choisit Joseph Dombey pour y participer :

Joseph Dombey, par son expérience et son inlassable curiosité d'homme des Lumières, joua un rôle fondamental dans cette mission scientifique, de 1778 à 1784, répondant à l'idéal de deux souverains - Louis XVI et Charles III - en faveur des progrès scientifiques et économiques des nations ${ }^{10}$.

8 Néanmoins, cette impulsion française à ce projet d'exploration, et notamment l'implication de Turgot décrite par Hamy ${ }^{11}$ et soutenue par Lang ${ }^{12}$, est remise en cause par l'Espagnol Enrique Álvarez López, qui signale qu'aucune preuve avérée ne confirme cette version. Certes, Turgot imagine bien une expédition vers des terres exotiques, mais il pense plutôt à Madagascar ou Pondichéry ${ }^{13}$.

Quoi qu'il en soit, la mission franco-espagnole est bien organisée, et il est établi que Dombey arrive au Pérou en 1778 et qu'il se met tout de suite au travail. En 1779, il a « botanisé » une grande partie des environs de Lima et a en même temps rassemblé une belle collection de curiosités des " anciens péruviens ${ }^{14}$, comme il les appelait. Comme cela a été évoqué précédemment, cette collection rassemblée par Dombey n'est pas son objectif premier. Sa mission principale est avant toute chose une mission naturaliste, botanique et minéralogique ${ }^{15}$. Malgré tout, lors de la préparation de son voyage, on lui a tout de même souligné l'importance de rapporter des exemples d'antiquités pour connaître l'histoire des anciens peuples du Pérou ${ }^{16}$.

Les anciens habitans, avant qu'ils eussent connus les Européens, avoient mis en usage plusieurs terres pour leurs ustensiles et pour former des petites pagodes 
qu'ils vénéroient. Ces terres subsistent encore, vous ferez très bien d'en apporter des échantillons qui font faute dans les cabinets et qui méritent d'être connus ${ }^{17}$. malheureusement pas être établies d'une manière certaine. Plusieurs lettres que Dombey envoie en France pour raconter ses expériences ${ }^{18}$ donnent quelques renseignements contradictoires. Dans une de ces lettres, il déclare avoir découvert les pièces lors de fouilles à Pachacamac ${ }^{19}$, site archéologique près de Lima, mais dans une autre, il déclare les avoir achetées ${ }^{20}$.

Le 3 avril 1779, Dombey envoie à bord du navire Bueno Consejo un premier lot de caisses remplies de ses trouvailles: semences, plants, herbiers, vases, platine, etc. Il n'est pas possible d'affirmer avec certitude que les deux pièces mises en avant dans cet article font partie de cet envoi. Hamy semble le penser ${ }^{21}$. Peut-être n'y en a-t-il qu'une des deux, peut-être aucune. Toujours est-il que cet envoi suit un parcours semé d'embûches et que c'est un véritable petit miracle si une partie arrive en France comme prévu. En effet, le Bueno Consejo est capturé par un corsaire anglais avant d'arriver en Espagne, sa cargaison se retrouve alors mise en vente à Lisbonne. Les Espagnols peuvent heureusement racheter cinq caisses de Dombey sur les sept qu'il a envoyées. Mais avant de les rendre à leurs destinataires français, ils s'emparent des plus belles pièces qu'ils y trouvent. Finalement, une petite partie seulement de l'envoi de Dombey arrive en France ${ }^{22}$.

Après cette première débâcle et considérant que la situation internationale se dégrade fortement, Dombey prend la décision de ne plus rien envoyer tant qu'il restera en Amérique latine ${ }^{23}$. Il stocke donc toutes ses découvertes et les emballe minutieusement dans des caisses entreposées à Lima au fur et à mesure de ses trouvailles. Quand vient le temps pour lui de rentrer en 1784 , il possède 73 caisses, qu'il fait charger sur le navire El Peruano qui fait le voyage Lima-Cadix. C'est ainsi que Dombey quitte définitivement le Pérou le 14 avril 1784. Pendant une longue escale à Rio de Janeiro ${ }^{24}$, Dombey explore un peu le Brésil et ajoute cinq caisses de plus à son inventaire. Ils repartent en novembre 1784 pour finalement arriver à Cadix le 28 février 1785, soit plus de dix mois après le départ de Lima. Malheureusement, les ennuis ne sont pas finis pour Dombey, ils ne font même que commencer.

Il faut noter qu'avant le départ de la mission, les Espagnols avaient fait signer à Dombey des accords qui fixaient les modalités de partage des connaissances et des objets entre l'Espagne et la France ${ }^{25}$. Au moment de son arrivée à Cadix avec ses 78 caisses, Dombey rencontre plusieurs difficultés. En effet, dans sa correspondance, il affirme qu'il est persécuté depuis le début de son voyage par un dénommé Ortega qui voudrait s'approprier ses découvertes pour qu'elles profitent avant tout à l'Espagne ${ }^{26}$. Il se retrouve donc bloqué à Cadix, et ses caisses sont mises de côté. Pour ajouter à son malheur, Dombey ne reçoit aucun soutien de la France et se voit même ordonner de collaborer avec les Espagnols. C'est ainsi qu'il finit par accepter un partage complet de ses caisses ainsi qu'une promesse écrite de sa part de ne rien publier de ses découvertes avant que les Espagnols ne le fassent ${ }^{27}$. Il est ainsi bloqué à Cadix pendant dix mois, et ce n'est que le 13 octobre 1785 qu'il peut rentrer à Paris, avec seulement 36 de ses caisses $^{28}$.

Comme pour le premier envoi de 1779, il est impossible de dire si les deux pièces de cet article se trouvent dans une de ces 36 caisses. Dans tous les cas, il n'est pas aisé de juger lequel de ces deux envois a connu le parcours le plus rocambolesque et une fois encore,

Les Cahiers de l'École du Louvre, 12 | 2018 
il est important de souligner la chance d'avoir la possibilité d'admirer ces pièces au musée du quai Branly - Jacques Chirac de nos jours.

Une fois ces deux pièces arrivées en France, leur parcours ne s'arrête pas pour autant. En effet, elles sont tout d'abord déposées le 31 janvier 1786 par Dombey au Cabinet des Antiques du roi selon les accords de la mission. Un inventaire écrit de ce dépôt montre que la chemise "porte sur son catalogue le $\mathrm{n}^{\circ} 52$ et est étiquetée de sa propre main: Tunique d'une vierge ou vestale du temple de Pachacamac ${ }^{29}$. "

Mais quelle place occupent ces deux objets dans la collection du Cabinet des Antiques parmi des pièces uniques de grande valeur, des trésors, des bijoux, des médailles et des antiquités égyptiennes et classiques? Il faut distinguer, parmi les consignes sur la collecte d'antiquités reçues par Dombey lors de la préparation de son voyage, celles données par l'abbé Barthélemy, alors responsable du Cabinet des Antiques du roi. L'abbé Barthélemy est un personnage clé, qui pose un regard précurseur sur les objets antiques des civilisations disparues. Ainsi, il est l'un des premiers à développer « une approche véritablement archéologique et non plus esthétique ${ }^{30}$ » de ces objets, la valeur de ces objets résidant selon lui dans le fait qu'ils permettaient de se faire une idée générale sur un peuple ancien. Pour lui, les deux pièces de Dombey ont tout à fait leur place dans le Cabinet des Antiques du roi, car il est "possible d'y trouver quelques traits de l'histoire de ces peuples ou de leur mythologie ${ }^{31}$ ».

Après la Révolution française, ces objets subissent plusieurs changements. D'un point de vue géographique tout d'abord, les objets sont déménagés vers la Bibliothèque nationale, comme le relève Hamy :

Le 20 messidor an III (8 juillet 1795) le conservateur du Dépôt de la rue de Beaune, le citoyen Naigeon, envoyait à la Bibliothèque de nombreux objets d'archéologie et d'ethnographie, parmi lesquels se distinguaient quelques vases péruviens provenant du comte de la Billarderie d'Angeviller, qui les avait reçus de Joseph Dombey, au retour de la mission ${ }^{32}[. .$.$] .$

Dans un second temps, le regard porté sur eux change également. En effet, « après la transformation révolutionnaire du Cabinet du roi et du Jardin des plantes en Muséum d'Histoire Naturell ${ }^{33}$ ", toutes les collections non naturalistes doivent être transférées dans un musée nouvellement créé, le Muséum des antiques, installé dans le bâtiment de la Bibliothèque nationale. Cette séparation entre collections naturalistes et collections d'antiquités traduit une rupture entre Artificialia et Naturalia, deux concepts liés qui étaient jusque-là à la base de l'organisation des cabinets de curiosités. Ainsi, les objets de Dombey sont séparés des collections naturalistes et rattachés aux antiquités classiques. Tous les objets se retrouvant dans le Muséum des antiques vont alors faire partie d'un projet comparatiste entre antiques et exotiques, et c'est ainsi que la chemise et le vase de Dombey obtiennent la même place que les antiquités égyptiennes, étrusques, grecques, romaines, etc. En effet, « le projet du "Muséum des Antiques" vise à unifier des objets parsemés à travers différentes collections publiques dont des artefacts exotiques ${ }^{34}$ ", mais malheureusement, «son échec suspend le projet original d'une connaissance des sociétés humaines par le biais de leurs cultures matérielles ${ }^{35}$ ".

Près d'un siècle plus tard, la chemise, le vase anthropomorphe et les autres pièces rapportées par Dombey en France sont déposés au musée d'ethnographie du Trocadéro lors de sa création en 1878, quand celui-ci recueille entre autres les collections conservées à la Bibliothèque nationale.

En 1897, ces deux pièces font partie d'une sélection d'objets que Hamy publie dans son livre Galerie américaine du Musée d'Ethnographie du Trocadéro. Il s'attache à décrire ces 
objets du point de vue de leur production matérielle et de leur utilisation, sans aucun jugement esthétique ni aucune référence artistique. À cette époque, l'étude et la classification de ce type d'objets doivent permettre d'obtenir un panorama de l'ensemble de l'activité humaine et de ses différents grades d'évolution.

Par la suite, en 1938, le musée du Trocadéro se transforme et devient le musée de l'Homme, et les deux pièces prennent leur nouvelle place au Palais de Chaillot. Dans ce nouveau cadre muséal, une grande exposition intitulée Chefs-d'œuvre de l'Amérique précolombienne ${ }^{36}$ est inaugurée en 1947 et présente une synthèse de l'art précolombien. Dans cette exposition se manifeste un important changement concernant le statut attribué aux objets archéologiques provenant des cultures non occidentales. Cette nouvelle approche approfondit les bases déjà proposées dans la grande exposition organisée par Georges Henri Rivière en 1928, Les arts anciens des Amériques, lors de laquelle ces pièces archéologiques furent pour la première fois regardées en tant qu'œuvres d'art.

Le choix des deux pièces de Dombey et de l'ensemble du corpus de pièces d'exposition tente d'atteindre un double objectif : non seulement « montrer ce qu'il y a de plus beau, du point de vue artistique, dans cet art indigène ", mais aussi " donner une impression de la multiplicité des grandes civilisations qui ont existé dans les Amériques avant leur découverte par les Européens ${ }^{37}$.

Pour atteindre ces deux objectifs, l'exposition est organisée de manière à présenter alternativement deux types de vitrines. D'une part, des vitrines consacrées chacune à une thématique précise, dans lesquelles l'aspect artistique des pièces est mis en avant ; d'autre part, des vitrines consacrées chacune à une culture spécifique, montrant la multiplicité des civilisations d'Amérique latine. Dans ce contexte muséographique, la chemise de Dombey est exposée dans une vitrine thématique centrée sur le tissu, où elle occupe une place privilégiée, tandis que son vase anthropomorphe est présenté dans une vitrine consacrée à la civilisation inca, d'une manière un peu plus anonyme, partageant l'espace avec de nombreuses autres pièces. Ces deux types de présentation se retrouvent dans les deux types de vitrine de l'exposition. Les vitrines consacrées aux civilisations sont riches en pièces et en exemples de leur mode de vie et de leurs pratiques culturelles, tandis que les vitrines thématiques à vocation artistique ne présentent que peu de pièces mais d'une grande valeur esthétique.

Par la suite, elles sont présentées dans l'exposition Ancien Pérou : vie pouvoir et mort organisée par Marie-France Fauvet-Berthelot et Danièle Lavallée au musée de l'Homme en 1987. Cette exposition présente plus de 680 pièces suivant un parcours thématique. Dans la vitrine "Textiles et costumes ", la chemise est exposée avec d'autres textiles (coiffes, sacs) pour former un ensemble vestimentaire complet. D'autres pièces en céramique qui représentent des personnages habillés en costumes traditionnels complètent la composition. Dans la vitrine dédiée à l'« Agriculture andine », le vase inca est exposé sur un piédestal le mettant en valeur. D'autres pièces en céramique et en bois, des reproductions de scènes de Guamán Poma de Ayala et des photos de terrasses agricoles dans les Andes l'accompagnent.

Dans cette exposition, la muséographie est centrée sur un objectif beaucoup plus pédagogique, avec l'utilisation de plusieurs types de supports pour faciliter la compréhension du discours. Les vitrines évoquent ainsi la production des pièces, leur utilisation dans la vie courante, et la diversification des techniques de production. 
Comme il a été mentionné précédemment, la troisième pièce étudiée dans cet article le vase phytomorphe à anses du Pérou - a été rapportée en France par le naturaliste Alcide d'Orbigny. Ce dernier est envoyé en Amérique du Sud pour une mission scientifique d'exploration et de collecte. Cette mission est l'une des premières organisées après l'indépendance du Pérou. La vice-royauté, colonie espagnole, n'existe plus et les institutions françaises n'ont plus besoin de demander l'aval de l'Espagne comme ce fut le cas pour la mission de Dombey. L'intérêt pour ces territoires s'en trouve accru et certaines institutions scientifiques expriment leur volonté d'y organiser des expéditions, comme le fait le Muséum d'histoire naturelle :

Parmi les divers pays, encore en assez grand nombre, qu'il serait important d'explorer dans l'intérêt de l'histoire naturelle, le Pérou et le Chili peuvent sous tous les rapports être placés au premier plan ${ }^{38}$.

C'est ainsi, qu'en 1825, le Muséum d'histoire naturelle organise une mission en Amérique du Sud, plus particulièrement en Bolivie et au Pérou, avec comme objectif de poursuivre vers le sud le voyage américain d'Alexander von Humboldt et d'Aimé Bonpland. Le Muséum choisit début novembre 1825 le jeune naturaliste Alcide d'Orbigny pour mener à bien cette mission. Il reçoit alors le titre de «voyageur naturaliste du Muséum d'Histoire Naturelle ».

Pour préparer au mieux son voyage, d'Orbigny réussit à imposer un délai de quelques mois. Ainsi, de novembre 1825 à juin 1826, il se forme à différents champs d'étude comme la zoologie, l'anatomie ou la géologie - pour laquelle il reçoit des leçons particulières d'Alexandre Brongniart, membre du Muséum, qui met aussi ses collections à la disposition du voyageur. Il souhaite également approfondir ses connaissances en géographie, ethnologie et histoire :

Je sentais que, devant voyager seul, il m'était indispensable de m'occuper de tout ce qui pouvait rendre plus complètes et moins arides des recherches de la nature de celles que j'allais entreprendre; je veux dire des sciences accessoires, comme la géographie, l'ethnologie, l'histoire, etc. Je visitai, dans cet intérêt, nos plus célèbres voyageurs. M. de Humboldt eut la complaisance de me poser une foule de questions à résoudre, et de me mettre au fait des moyens d'observation dans ces contrées lointaines ${ }^{39}$.

D’Orbigny reçoit des instructions précises pour bien accomplir sa tâche. Ainsi, il écrit : M. Isidore Geoffroy Saint-Hilaire vint rédiger pour moi, dans les galeries du Muséum, les notes les plus précieuses, sur les objets à recueillir en mammifères et en oiseaux; et, spécialement, sur les observations à faire relativement à leurs mœurs, encore si peu connues ${ }^{40}$.

Cependant, ces instructions précises concernent surtout la botanique, la zoologie et la minéralogie. Pour l'archéologie et l'ethnologie, il en est tout autre. Pascal Riviale mentionne que «les recommandations d'ordre archéologique et anthropologique étaient extrêmement succinctes comme on en jugera: "Rechercher les traces, s'il en existe encore, des monuments des anciens péruviens tels que bâtiments, temples, idoles, statues, monnaies [sic], tombeaux, instruments." ${ }^{41}$ ».

Il faut tout de même signaler que, profitant de cette opportunité, Alexandre Brongniart, directeur du musée de Sèvres, demande au jeune naturaliste de rassembler une collection de pièces en céramique pour enrichir les collections du musée. Brongniart a rédigé en 1824 des « instructions générales sur la manière de concourir à compléter la collection relative aux arts céramiques et de la vitrification établie à la Manufacture royale de porcelaine à Sèvres ${ }^{42}$ " à donner aux voyageurs et expéditionnaires qui partaient dans différents coins du monde. Ces instructions sont 
détaillées et complétées selon la mission ou le voyage et surtout en fonction des particularités des pays :

Je l'ai prié de recueillir pour les collections de céramiques de Sèvres toutes les pièces de poteries qui pourraient nous instruire sur l'état des arts céramiques, tant chez les indigènes avant la Conquête que dans les temps modernes ${ }^{43}$.

Mais les instructions de Brongniart concernent davantage la technique de fabrication, les matériaux, la cuisson et le moulage que la valeur archéologique ou ethnologique des pièces.

Cette mission était prévue pour une durée de trois ans mais d'Orbigny reste en Amérique du Sud pendant sept ans, de 1826 à 1833. Tout d'abord, il arrive sur le continent sud-américain du côté de l'Atlantique. Il visite la rivière du Paraná, différentes provinces d'Argentine, puis le sud du Paraguay et le nord de la Patagonie. Il reste dans cette zone pendant plus de trois années. En juillet 1829, d'Orbigny fait un premier envoi au musée de Sèvres, composé de vases et de coupes provenant du Paraguay et d'Argentine. Cet envoi est inventorié par le musée une première fois dès sa réception, mais il existe un second inventaire, réalisé quelques années plus tard, qui regroupe l'ensemble des objets collectés par d'Orbigny. Cependant, ces deux inventaires sont différents. Certaines pièces du premier inventaire n'y figurent plus, et les raisons de ces disparitions ne nous sont pas parvenues. Casse? Perte? Vol? Récupération personnelle?

En 1830, d'Orbigny poursuit son voyage et arrive finalement du côté Pacifique de l'Amérique du Sud. Il entre d'abord en Bolivie, puis au Pérou. Concernant les pièces archéologiques qu'il rapporte de ces deux pays, il est intéressant de signaler qu'à maintes reprises, d'Orbigny se lance dans des fouilles de tombes préhispaniques, mais qu'il est très souvent déçu car il n'y trouve rien. Malgré tout, il parvient régulièrement à acheter quelques pièces auprès de la population locale ${ }^{44}$.

À son retour en 1834, d'Orbigny rapporte une collection de vases provenant principalement de Bolivie et du Pérou. Contrairement aux pièces qu'il envoie en 1829 et dont il fait don au musée de Sèvres, il propose cette fois-ci cette collection à la vente à Brongniart, qui demande l'aide financière du roi :

Je ne peux que le prier obtenir du roi un crédit extraordinaire de $1200 \mathrm{fr}$ pour acheter à M. Dessaline D'Orbigny un choix de poteries de l'Amérique du Sud pour être placées dans la collection céramique de la Manufacture Royale de Porcelaine ${ }^{45}$.

Brongniart ne parvient pas à acheter la collection entière rapportée par d'Orbigny ${ }^{46}$. Il doit faire une sélection parmi les pièces :

J'ai fait un choix d'environ 20 pièces caractérisées par les diverses considérations que je viens d'exposer et j'ai l'honneur de vous proposer de demander au Roi d'en faire l'acquisition pour le Musée céramique de la Manufacture ${ }^{47}$.

La pièce rapportée par d'Orbigny qui est étudiée dans cet article provient de cette deuxième collection, vendue au musée de Sèvres en 1834 .

Elle est mentionnée pour la première fois dans une liste récapitulative que rédige Brongniart lors de sa sélection en mars 1834 et qui s'intitule « Liste de vases antiques de l'Amérique Méridionale que Mr. Brongniart a mis à part ». Le vase porte le numéro 17 et est décrit sous ces termes :

17. Vase noir avec arabesque, rencontré dans les tombeaux des environs de Lima (Pérou) ${ }^{48}$.

Par la suite, A. Brongniart et D. Riocreux (conservateur des collections au musée de Sèvres) présentent cette pièce dans leur ouvrage intitulé Description méthodique du Musée 
céramique de la Manufacture Royale de Porcelaine de Sèvres ${ }^{49}$, dans la partie dédiée aux terres cuites du Pérou, époque antique :

Bouteille en forme de baril, ornée de méandres et de dents de loup gravés profondément [...]; pâte noirâtre matte. Long. 0,18c . - Tirés des tombeaux des Indiens Quichuas de Lima. (Par M. A. d'Orbigny, 1834) ${ }^{50}$.

Dans le même ouvrage, un dessin à main levée de la pièce est présenté dans la planche XVIII avec d'autres exemples de poteries péruviennes antiques ${ }^{51}$.

Enfin, en 1956, le catalogue de François Reyniers sur la collection américaine du musée de Sèvres, intitulé Céramiques américaines du Musée National de Céramique de Sèvres. Inventaire des Collections publiques françaises, référence notre pièce avec le numéro 194 et la mention suivante :

194. Baril à deux anses. Couleur noire. Décor gravé. Haut. 0,$14 ;$ long. 0,20; ép. 0,10.

Inventaire 1765. Don. A. d'Orbigny. Entré en $1834^{52}$.

Il est intéressant de noter que Reyniers spécifie que la pièce a été trouvée par d'Orbigny près de Lima en 1827. Or, cette date et ce lieu ne correspondent pas du tout à l'itinéraire suivi par d'Orbigny.

Le vase de d'Orbigny est choisi pour sa spécificité technique et sa valeur archéologique par Brongniart qui veut, en accord avec la ligne directrice du musée céramique de Sèvres, former une collection provenant de toutes les régions du monde et de toutes les époques pour avoir un inventaire technique complet de la production de céramique.

Il ne s'agit pas d'établir la valeur commerciale de ces objets mais de contribuer à compléter l'histoire des arts céramiques ${ }^{53}$.

Ainsi, les objets qui forment la collection du musée de Sèvres, à laquelle appartient le vase de d'Orbigny, n'ont qu'une valeur historique et esthétique secondaire. Les références documentaires précédemment citées montrent bien que cette considération principalement technique de la pièce reste constante.

21 La quatrième pièce mise en avant dans cet article - un masque de pierre sculptée provenant du Mexique - a été rapportée en France par l'explorateur franco-belge Auguste Génin à la fin du XIX ${ }^{e}$ siècle. Il est très intéressant de suivre cet explorateur car il nous révèle les pensées et les méthodes scientifiques de la fin du XIX ${ }^{e}$ siècle, soit un siècle après Dombey et un demi-siècle après d'Orbigny. De plus, le théâtre des explorations de Génin se situe au Mexique.

Sa première expédition date de 1879 , quand il doit se rendre au Mexique pour reprendre les affaires de son père tout juste décédé. Comme il le mentionne dans une de ses lettres: «Pendant dix ans, de 1879 à 1889, j'eus l'occasion de parcourir la République Mexicaine en tous sens ${ }^{54}$.» C'est pendant cette période qu'il réalise ses premières excursions archéologiques à Teotihuacan et à Cholula. Par la suite, il continue ses travaux à travers le Mexique (Querétaro, Guanajuato, San Luis Potosi, Yucatan, Oaxaca, etc.). Son intérêt pour les explorations sur le terrain s'accorde parfaitement avec son goût et son esprit de collectionneur et lui offre par exemple l'opportunité d'acheter une collection assez importante appartenant au père Francisco Plancarte, archéologue et collectionneur reconnu, à la mort de celui-ci.

En 1892, Génin présente une demande d'autorisation au ministère de l'Instruction publique pour réaliser une mission au Mexique. Cette demande est appuyée par Hamy qui lui accorde une confiance totale. Par arrêté du 14 janvier $1892^{55}$, il est nommé à la tête d'une mission scientifique gratuite au Mexique "à l'effet d'y poursuivre des recherches relatives à l'archéologique, à l'ethnographie, à la linguistique [...] étudier 
l'état de l'instruction publique dans ce pays ainsi que les méthodes d'enseignement qui y sont employées ${ }^{56} »$.

Par ailleurs, Génin doit aussi s'occuper de la situation des collections rassemblées par Désiré Charnay, qui restaient au Mexique sans pouvoir être envoyées en France. Le ministère de l'Instruction publique lui avait demandé de faire les démarches nécessaires :

Je devais, de plus, profiter de mon séjour au Mexique pour demander au Gouvernement Mexicain, d'une façon officieuse, la solution qu'il pensait donner aux demandes de M. Désiré Charnay relativement à ses collections détenues par une application rigoureuse de la loi qui prohibe l'exportation des objets provenant de fouilles ${ }^{57}$.

Génin reçoit aussi des instructions de la part de Hamy, comme il le signale :

Enfin, sur les indications de M. le Docteur Hamy, m'occuper de la régularisation du service des publications mexicaines données en échange au corps savants [sic] français ${ }^{58}$.

23 Le masque anthropomorphe en pierre dure sculptée collecté par Génin provient d'une série d'explorations effectuées en 1893 à Yahualica, un village de l'État d'Hidalgo, plus précisément dans « la sierra de Huachinango et les pays de Tecolutla et Metlaltoyuca ${ }^{59}$ " car cette région était "couverte de monuments anciens qui n'ont pas encore été explorés $^{60}$ \%. Dans ce même temps, arrive à Yahualica «une commission du gouvernement de l'État d'Hidalgo pour faire des fouilles dans les tumulus des environs ${ }^{61}$ ». À la tête de cette commission était Louis A. Escandón, qui autorise Génin à participer à ces recherches. Ce masque fait partie des cinq objets que Génin obtient lors de ces fouilles ${ }^{62}$.

Pendant cette mission, il rassemble en outre diverses collections : un herbier mexicain, une collection d'insectes et de reptiles, une collection d'ornithologie, une collection d'ossements et de crânes, une collection d'archéologie et d'ethnographie. Son premier envoi en France date de 1893. Il envoie également une collection botanique et quelques manuscrits mexicains achetés aux héritiers de l'explorateur français Paul Maury, mort au Mexique en $1893^{63}$, ainsi qu'une grande quantité de publications mexicaines sur différents domaines du savoir.

Il faut noter que Génin se trouve confronté au même problème qu'avait rencontré Charnay quelques années auparavant, à savoir l'interdiction officielle de faire sortir des pièces archéologiques du Mexique ; cela l'empêche de récupérer les collections de son prédécesseur ${ }^{64}$. Mais, contrairement à Charnay, Génin réussit à contourner cette difficulté et à envoyer en France de nombreuses caisses remplies d'antiquités :

Les caisses contenant les résultats de mes fouilles ont heureusement passé la frontière - malgré les dispositions prohibitives du Ministère des Finances, que cela regarde on ne sait trop pourquoi ${ }^{65}$.

24 Les méthodes utilisées pour parvenir à ses fins restent floues, mais il parle à plusieurs reprises dans ses écrits de ses bons contacts avec le président du Mexique et avec le ministre de l'Instruction publique, qui acceptent d'autoriser officieusement l'envoi de Génin, pourvu que tout ceci n'attire pas l'attention des archéologues mexicains. Nous ne savons malheureusement pas comment Génin a obtenu une telle mansuétude de la part des autorités mexicaines.

Je suis à peu près certain que les objets dignes d'être transportés en France, que je trouverai ne seront pas arrêtés à la Douane : on ne me donnera aucune autorisation mais on fermera les yeux. Je crois pouvoir profiter, sans faillir en rien, de cette tolérance ${ }^{66}$. 
J'ai eu le plaisir de causer longuement avec M. le Président de la République et M. Baranda, Ministre de l'Instruction publique; ils ont bien voulu me promettre de me laisser emporter sans difficulté le produit de mes fouilles mais tout ceci officieusement afin d'éviter les criailleries des archéologues du $\mathrm{cru}^{67}$.

Les collections rassemblées par Génin rejoignent le fonds du Muséum d'histoire naturelle, de la Bibliothèque nationale et du musée d'ethnographie du Trocadéro. Ce dernier reçoit deux cent cinq lots d'objets divers, archéologiques et ethnographiques ${ }^{68}$ (collections enregistrées 71.1897.52 et 71.1897.53), dont le masque de pierre étudié ici.

Cette collaboration étroite avec le musée du Trocadéro continue et Génin, ayant toujours le désir de compléter les collections mexicaines en France, envoie en 1922 sa plus grande donation (71.1924.13), malgré la difficulté à exporter le matériel archéologique mexicain.

De la même façon, Génin fait bénéficier de ses découvertes différents pays avec qui il maintient un lien scientifique et personnel, comme la Belgique. Ainsi, en 1923 et en 1930, il fait d'importantes donations aux musées du Cinquantenaire ${ }^{69}$.

Pendant les années 1930, cet objet est probablement exposé dans une des salles dédiées au savant, au sein de la galerie Auguste Génin B6 dans la section américaine de l'exposition permanente du musée du Trocadéro. Malheureusement, il n'existe pas d'informations détaillées sur les objets qui y sont présentés, mais, si le masque en fait partie, il est probablement peu visible, dans une vitrine surchargée.

Par la suite, la pièce est présentée dans deux grandes expositions organisées à Paris. La première exposition, Les arts anciens de l'Amérique, est organisée par Georges-Henri Rivière au musée des Arts décoratifs en 1928. Comme il a été expliqué précédemment, c'est la première grande exposition en France dédiée exclusivement aux civilisations américaines, et dans laquelle l'aspect esthétique des pièces est mis en avant. Le masque de Génin fait partie d'un groupe d'objets prêtés par le musée du Trocadéro et spécialement choisis sur des critères esthétiques :

Le Dr Rivet, directeur du Musée d'Ethnographie du Trocadéro, a consenti à se

dessaisir d'un grand nombre des plus belles pièces de ce Musée ${ }^{70}$.

La pièce correspond au numéro 134 du catalogue de l'exposition.

La deuxième exposition dans laquelle ce masque est présenté est Chefs-d'œuvre de l'Amérique précolombienne, organisée par le musée de l'Homme en 1947, exposition présentée précédemment. La pièce correspond au numéro $7 \mathrm{du}$ catalogue de l'exposition:

7. Masque en marbre verdâtre : face humaine au nez fin, bouche ouverte, oreilles stylisées. Partie postérieure encadrée de deux bandes portant deux perforations.

Hauteur : $13 \mathrm{~cm}$ 2. Largeur : $14 \mathrm{~cm} 3^{71}$.

À l'exemple du masque de Génin, l'exposition de 1947 reprend la majeure partie des pièces du musée du Trocadéro présentes dans l'exposition de 1928, et ce, avec l'objectif clair de poursuivre l'approche esthétique des pièces archéologiques développée en 1928. Comme cela a été dit à propos des pièces de Dombey, dans l'exposition Chefsd'œuvre de l'Amérique précolombienne de 1947, une partie des vitrines présentées au public est organisée selon une thématique précise et avec une vocation artistique. C'est dans une de ces vitrines, intitulée "Masques », qu'est exposé le masque de Génin avec quelques autres objets du même type.

Enfin, la cinquième pièce mise en avant dans cet article, le masque funéraire en or Nasca, est arrivée en France en 1930 grâce au collectionneur péruvien Guillermo Schmidt-Pizarro. Ainsi, la pièce n'est plus rapportée par un explorateur ou un 
botaniste, contrairement à ce qui a été présenté précédemment. Ce changement notable caractérise une nouvelle période, le début $\mathrm{du} \mathrm{xx}^{\mathrm{e}}$ siècle, et une autre façon d'envisager et de concevoir les collections d'objets archéologiques et ethnographiques.

Guillermo Schmidt-Pizarro est un collectionneur péruvien dont l'histoire est quelque peu mystérieuse, car peu de détails sont connus sur sa vie et sur la façon dont il a rassemblé ses collections archéologiques. Il est né vers 1881, mais les traces de son activité de collectionneur n'apparaissent qu'à partir des années 1930, date à laquelle il se met à donner et à vendre de nombreuses pièces à toutes les principales institutions muséales du monde entier. Ainsi, Schmidt-Pizarro vend des objets péruviens au British Museum, au gouvernement espagnol, au musée d'ethnographie du Trocadéro et à plusieurs institutions aux États-Unis (Copper Hewitt Museum, Metropolitan Museum, National Museum of the American Indian). Sa vente la plus importante est réalisée en Espagne: le 3 décembre 1930, l'État espagnol fait l'acquisition d'une collection ( 42 pièces de textiles précolombiens et 6 pièces de textiles coloniaux) pour le Musée archéologique national pour 6100 pesetas $^{72}$.

S.A.S. le Roi a pris les dispositions suivantes: $1^{\circ}$ que la liste d'objets incasiques proposés par M. Schmidt Pizarro soit acquise pour le Musée d'Archéologie National ${ }^{73}$.

Cette riche collection de textiles est transférée au musée d'Amérique de Madrid lors de sa création en 1941. De plus, Schmidt-Pizarro vend en 1933 une collection d'une vingtaine de pièces au British Museum, principalement des tissus précolombiens.

Cependant, le point le plus important ici est la relation étroite qu'il entretient avec le musée d'ethnographie du Trocadéro. Guillermo Schmidt-Pizarro rencontre GeorgesHenri Rivière au musée du Trocadéro le 26 juillet 1930 pour présenter une collection destinée à la vente composée de 21 pièces archéologiques péruviennes en pierre, en tissu, en métal, etc.

Georges-Henri Rivière, dans une lettre du 28 juillet 1930 envoyée à Schmidt-Pizarro, déclare l'acceptation de l'achat de cette collection au prix de $25000 \mathrm{~F}^{74}$. Le paiement est effectué en deux fois, un premier versement de $5000 \mathrm{~F}$ par chèque le 27 juillet 1930 et un deuxième de $20000 \mathrm{~F}$ en novembre 1930. Dans cette lettre, Rivière explique qu'il va essayer que ce montant de $25000 \mathrm{~F}$ soit pris en charge par les bienfaiteurs du musée, mais que si cela n'est pas possible il assumera lui-même cette dépense ${ }^{75}$. Par la suite, en octobre 1930, Schmidt-Pizarro réalise une donation de onze pièces archéologiques péruviennes au musée du Trocadéro.

L'objet mis en avant dans ce travail fait partie de la vente effectuée par Schmidt-Pizarro en juillet 1930. C'est une pièce exceptionnelle qui explique à elle seule l'intérêt de Rivière et la somme importante qu'il est prêt à payer, puisant même dans ses fonds personnels.

Après son entrée au musée, la pièce est présentée dans deux expositions importantes. En 1947, dans l'exposition Chefs-d'œuvre de l'Amérique précolombienne, elle est exposée dans la partie centrale de la vitrine "Métaux » avec d'autres objets de petite taille. Comme cela a déjà été mentionné, deux sortes de vitrines se côtoient dans cette exposition. La vitrine des objets en métal est naturellement une vitrine à vocation esthétique, et le masque en or de Schmidt-Pizarro en est la pièce phare.

En 1987, la pièce est présentée dans l'exposition Ancien Pérou : vie, pouvoir et mort, dans la vitrine "Images et insignes du pouvoir ", aux côtés de différentes pièces en métal, en tissu et en plumes. Comme pour les deux pièces de Dombey, le masque en or est choisi 
avec l'objectif de clarifier le discours scientifique de l'exposition. Il s'agit donc d'un objet appuyant et illustrant un discours et non plus un objet générateur d'un imaginaire sur l'Autre et l'Ailleurs.

31 Ainsi, après la description de ces cinq pièces importantes, qui aident à clarifier l'histoire de la formation des collections archéologiques latino-américaines, et la présentation des personnages qui ont permis que ces pièces arrivent en France, nous pouvons maintenant analyser la façon dont ces collections ont été formées et utilisées pour comprendre et expliquer ces cultures lointaines. Ces questions sont fondamentales dans ce sujet de recherche pour comprendre la pensée de l'époque sur l'Autre et l'Ailleurs.

Tout d'abord, il faut souligner que, jusqu'à la fin du xIX siècle, la formation des collections archéologiques et ethnographiques provenant d'Amérique latine est très liée à la recherche ainsi qu'à l'accroissement des collections d'histoire naturelle. Les collections rapportées par Joseph Dombey et Alcide d'Orbigny en sont le parfait exemple. Pascal Riviale confirme ce phénomène en affirmant que «c'est bien dans le domaine de l'histoire naturelle que nous devons nous placer pour évoquer la curiosité américaine. Les sondages [...] effectués [...] entre 1740 et 1792, nous montrent clairement l'association étroite entre ethnographie "exotique" et histoire naturelle ${ }^{76}$ ». En effet, à cette époque, l'histoire naturelle est la science principale et les autres disciplines, comme l'archéologie et l'ethnographie, progressent d'une façon annexe et secondaire.

Ensuite, à la fin du XIX siècle et au début du xxe siècle, la situation change à mesure que les disciplines scientifiques considérées comme secondaires à leur apparition se spécialisent et prennent leur indépendance. Une profusion de missions spécialisées dans ces domaines avec des objectifs scientifiques précis accélèrent fortement la formation des collections ${ }^{77}$. L'exemple d'Auguste Génin permet d'observer l'éclosion de ces nouvelles disciplines scientifiques. En effet, au cours de ses premières missions, Génin poursuit des objectifs archéologiques et ethnographiques, sans oublier toutefois de rassembler des collections naturalistes. Mais, au fil du temps, il est possible d'observer une évolution vers un travail et une collecte exclusivement archéologique et ethnographique. Enfin, au cours $\mathrm{du} \mathrm{xx}^{\mathrm{e}}$ siècle, les recherches archéologiques et ethnographiques se consolident fortement et rassemblent d'importantes collections pour la France. Cette évolution est également impulsée par la création de l'Institut d'ethnologie en 1925 et par l'arrivée de Paul Rivet à la tête du musée d'ethnographie du Trocadéro. Dans ce contexte, le premier projet qui permet à l'ethnographie de s'affirmer comme une discipline à part entière et qui met en œuvre une collecte d'objets de manière plus scientifique est la mission " Dakar-Djibouti ${ }^{78}$ ».

Deuxièmement, comme nous l'avons montré précédemment, l'intervention de certains personnages (explorateurs, naturalistes, archéologues et collectionneurs) est décisive dans la formation des collections provenant d'Amérique latine. On observe que leur formation se spécialise, et que leurs objectifs se précisent. Ils sont, au début, issus de domaines très variés, et reçoivent comme Joseph Dombey des instructions très générales qui leur permettent de choisir eux-mêmes les objets sur le terrain. Ensuite, les commanditaires deviennent de plus en plus exigeants concernant la formation de leurs collections, et transmettent des instructions bien plus spécifiques et détaillées. Il est important de noter que ces commanditaires profitent la plupart du temps de toute l'infrastructure de l'expansion coloniale de l'époque pour se faire rapporter de 
nouveaux objets « exotiques » prélevés et collectés directement sur le terrain par des explorateurs, des voyageurs, des ambassadeurs, des fonctionnaires et des membres du personnel des nouvelles administrations coloniales. Pour optimiser cette collecte, « les acteurs de la recherche avaient à leur disposition une grande diversité de supports institutionnels - officiels ou non [...]. Parmi les supports officiels on retiendra ici surtout le rôle joué par le Muséum d'histoire naturelle et le Service des missions scientifiques et littéraires ${ }^{79}$ ». Dans ce contexte, les indications que d'Orbigny reçoivent de la part d'Alexandre Brongniart, directeur du musée de la manufacture de Sèvres, sont un exemple parfait.

Progressivement, les explorateurs se spécialisent dans le domaine de la recherche de collections archéologiques et ethnographiques en participant aux fouilles archéologiques, comme le fait Auguste Génin. À partir du xxe siècle, le contexte change, et avec l'influence de la vision artistique sur ce type de collections, un commerce spécialisé émerge au sein d'un marché de l'art de plus en plus conséquent et dynamique. L'accroissement des collections dans les institutions muséales se fait non seulement par l'organisation des missions scientifiques qui rapportent d'importantes collections mais aussi par l'achat, la donation et les legs. Dans ce cadre, SchmidtPizarro est un bon exemple.

Troisièmement, le statut attribué à ces collections provenant d'Amérique latine et aux objets proprement dits évolue lui aussi au fil du temps. Avant la Révolution française, le statut de ces objets, principalement rassemblés dans les cabinets de curiosités et mélangés avec les objets naturalistes (Artificialia et Naturalia côte à côte), est avant tout conféré par leur caractère particulier et unique. À la fin de cette période apparaissent d'autres manières de les voir, dépassant la simple curiosité européenne, à l'image des idées novatrices de l'abbé Barthélemy : comme le dit Irène Aghion, il ne s'agit plus pour lui de « les regarder comme des pièces rares et étranges, mais de les replacer dans leur contexte géographique et d'en comprendre la fonction ${ }^{80} »$.

Après la Révolution, « les objets manufacturés par l'homme se séparent des collections naturalistes ${ }^{81}$ » et comme "l'unité entre Artificialia et Naturalia qui régissait les collections depuis la Renaissance est rompue ${ }^{82}$ ", cela "oblige, dans le découpage des collections, à donner une place aux objets à caractère ethnographique ${ }^{83}$ " dans un nouveau projet appelé « Muséum des Antiques ${ }^{84}$ ». Ce projet devient l'embryon d'un regard comparatiste dans lequel les objets latino-américains sont considérés de la même façon que les antiquités classiques ${ }^{85}$. Par la suite, l'influence de l'anthropologie physique et la prédominance des principes de classification fortement liés à l'histoire naturelle font des objets archéologiques et ethnographiques avant tout des artéfacts qui doivent apporter des informations techniques de fabrication et d'utilisation et ainsi permettre de comparer et de classifier les sociétés qui les produisent sur l'échelle de l'évolution humaine. Selon Pascal Riviale, cette démarche s'inscrit dans un grand mouvement de l'époque visant à " contribuer à un vaste "inventaire" de la planète, de ses êtres et de ses choses ${ }^{86}$ ». Et c'est dans des institutions telles que le musée d'ethnographie du Trocadéro ou son prédécesseur l'éphémère Musée ethnographique des missions scientifiques que ces idées et ces visions de l'objet atteignent leur plein essor.

Ainsi conçu, avec des séries non interrompues, le musée ethnographique ne sera pas une collection d'objets bizarres, étranges, quelquefois futiles, dispersés çà et là, mais une histoire des mœurs et des usages, histoire parlant aux yeux où, depuis les armes jusqu'aux vêtements, depuis les habitations jusqu'aux bijoux et aux meubles 
les plus grossiers, tout objet concourra à former cet ensemble de matériaux qui permettra d'établir des comparaisons illimitées entre les civilisations primitives des populations, existantes ou éteintes, du monde entier ${ }^{87}$. latine se développe à la fin du $\mathrm{XIX}^{\mathrm{e}}$ siècle. Cet intérêt ne se limite plus seulement à l'objectif de former une collection mais se manifeste également par une volonté d'étude, de recherche et de compréhension des cultures des Amériques; celle-ci se traduit par le mouvement de l'Américanisme, qui s'amplifiera et se renforcera encore tout au long du $\mathrm{xx}^{\mathrm{e}}$ siècle.

Un nouveau statut attribué aux objets archéologiques et ethnographiques fait alors son apparition dans le premier tiers $\mathrm{du} \mathrm{xx}^{\mathrm{e}}$ siècle, dont la parfaite illustration est l'exposition Les arts des Amériques présentée en 1928 par George-Henri Rivière au musée des Arts décoratifs, où pour la première fois en France ces objets sont principalement considérés comme des objets d'art. Dans ce contexte, le mouvement surréaliste a fortement aidé à développer cette nouvelle façon de considérer ces collections « pour la teneur expressive et pour la valeur esthétique ou poétique de ces objets ${ }^{88} »$. Il s'agit là d'une complète révolution vers un certain regard qui sera présent tout au long du $\mathrm{xx}^{\mathrm{e}}$ siècle. Si auparavant les collections archéologiques et ethnographiques étaient exclusivement liées à la science, et donc considérées comme des objets d'étude, elles relèvent désormais également de l'art et sont donc observées comme des œuvres d'art. La création du musée de l'Homme en 1937 propose une rupture avec l'approche faite par le musée d'ethnographie du Trocadéro. En effet, le musée de l'Homme est voulu comme un musée laboratoire et ses créateurs Paul Rivet et Georges-Henri Rivière avaient «la volonté de faire de [leur] musée le grand établissement d'enseignement populaire et de recherche scientifique qu'il doit être, de mettre en valeur toutes ses incalculables richesses et de le rendre digne de l'admirable effort colonial de [leur] pays $^{89} »$. Le statut esthétique des collections n'est pas mis de côté. Il complète le statut scientifique pour faciliter le discours et être plus facilement accessible au public. Cette thématique double se retrouve très clairement dans l'exposition de 1947, Chefs-d'œuvre de l'Amérique précolombienne, dans laquelle deux types de discours sont utilisés: scientifique et esthétique. Il est important de remarquer qu'à ces deux statuts donnés aux collections s'ajoute la dimension politique: les collections montrent la réussite colonialiste de la France.

Ainsi, dans ce panorama sur l'évolution des collections latino-américaines, il est possible d'observer la multiplicité des statuts que les objets ont pu acquérir au fil du temps. Ceux-ci ont été principalement définis par l'intervention d'acteurs majeurs et de mouvements scientifiques et artistiques qui ont joué un rôle important, ainsi que par les institutions muséales dans lesquelles les objets ont été placés. Il est important de noter que cette "muséalisation» prend de plus en plus d'importance pour la compréhension de l'objet et que le musée doit désormais être considéré comme un acteur clé dans sa "vie»; l'action du musée doit dorénavant être intégrée à la biographie de l'objet ${ }^{90}$.

Il faut mentionner que ces nouveaux statuts ne s'appliquent pas de manière générale dans tous les contextes car dans certaines sphères ou dans certaines institutions, ces objets reçoivent un statut spécial, conditionné à une vision ou à des objectifs précis. Dans ce cadre, il est clair que les objets que collecte d'Orbigny en suivant les instructions précises données par Brongniart sont choisis principalement pour les 
spécificités techniques de leur production céramique. Ainsi, le statut donné à ce type de collections au sein du musée de la manufacture de Sèvres reste subordonné aux aspects techniques de fabrication et de décoration.

Finalement, l'observation de la façon dont les collections d'objets provenant d'Amérique latine se sont développées, dont certains acteurs, comme ceux qui ont été mentionnés dans cet article, sont intervenus, et dont ces collections ont été utilisées (parcours, statut, échanges, achats, mise en exposition) permet de parvenir à un niveau de compréhension qui apporte un éclairage majeur sur la pensée des époques et des raisonnements engendrés par des événements politiques, scientifiques ou artistiques et qui aide à découvrir comment le regard sur l'Autre et sur l'Ailleurs a évolué.

Ainsi, à partir de l'étude de la qualification des objets archéologiques et ethnographiques provenant d'Amérique latine, c'est-à-dire à partir de la façon de « restituer la complexité des processus d'identification ${ }^{91}$ " pour ce type d'objets, établir une histoire des collections permet d'aller plus loin qu'une simple classification ou qu'une simple succession historique. Cela nous permet d'étudier les pensées et les raisonnements des acteurs et de différents mouvements (scientifiques, politiques, artistiques) et ainsi de comprendre l'évolution du regard sur l'Autre.

\section{NOTES}

1. De manière objective, cette richesse se concentre en majorité en île-de-France et c'est ainsi que mes investigations ont commencé dans les institutions franciliennes : musée du quai Branly Jacques Chirac, Musée de la céramique de Sèvres, musée de Saint-Germain-en-Laye et bibliothèque Sainte-Geneviève. Il a tout de même fallu définir poser quelques limites pour établir mon corpus d'étude et c'est ainsi que j'ai défini un ensemble d'environ 50000 pièces. C'est à partir de ce corpus que je me suis plongée dans l'histoire de chaque pièce pour établir une histoire de la formation des collections latino-américaines.

2. Pour approfondir ce sujet consulter Thierry Bonnot, La vie des objets. D'ustensiles banals à objets de collection, Paris, Maison des sciences de l'homme, 2002.

3. Igor Kopytoff, "The cultural biography of things: commoditization as process ", dans Arjun Appadurai (dir.), The social life of things. Commodities in cultural perspective, Cambridge, Cambridge university press, 1986, pp. 64-92.

4. Pour consulter la première description détaillée de la pièce voir: Ernest-Théodore Hamy, Galerie américaine $d u$ musée d'ethnographie du Trocadéro. Choix de pièces archéologiques et ethnographiques, Paris, Ernest Leroux, 1897, pp. 95-96.

5. Idem, Ibidem, p. 68.

6. Deux pièces ont été choisies pour le cas de Joseph Dombey, à la différence des autres personnages. La raison de ce choix est la relation très étroite entre ces deux pièces, un destin croisé qui ne les a quasiment jamais séparées depuis leur arrivée en France, depuis le Cabinet du roi jusqu'au musée du quai Branly - Jacques Chirac, et au cours de différentes expositions auxquelles elles ont participé.

7. E.-T. Hamy, op. cit. note 4, pp. 68, 95. 
8. E.-T. Hamy, Joseph Dombey, médecin, naturaliste, archéologue, explorateur du Pérou, du Chili et du Brésil (1778-1785) : sa vie, son œuvre, sa correspondance, avec un choix de pièces relatives à sa mission, Paris, E. Guilmoto, 1905, p. II-III.

9. Catherine Lang, "Joseph Dombey et l'expédition de Ruiz et Pavon: étude des itinéraires (1778-1784) », Bulletin de la société botanique de France. Lettres botaniques, vol. 132, n³ 3, 1985, p. 260.

10. Idem, Ibidem, p. 260.

11. E.-T. Hamy, op. cit. note 8, pp. XV-XVI.

12. C. Lang, op. cit. note 9, pp. 259-260.

13. Enrique Álvarez López, « Dombey y la Expedición al Perú y Chile », Anales del Instituto Botánico AJ Cavanilles, vol. 14, 1955, p. 36.

14. E.-T. Hamy, op. cit. note 8, p. 247 (Lettre au Comte d'Angeviller).

15. Un des objectifs assignés à Dombey était de remplacer les herbiers de Joseph Jussieu malheureusement perdus.

16. Jean-Jacques Barthélemy, CEuvres de J. J. Barthélemy, Paris, A. Belin, vol. 4, 1821, p. 434.

17. E.-T. Hamy, op. cit. note 8, p. 320 (Lettre Instructions de Séguier).

18. Lettres publiées en grande partie par E.-T. Hamy en 1905 dans op. cit. note 8.

19. Idem, Ibidem, p. 52 (Lettres à André Thouin).

20. Id., Ibid., p. 41.

21. Id., Ibid., p. XLVII.

22. Id., Ibid., p. L.

23. Id., Ibid., p. 56 (Lettres à André Thouin).

24. La traversée du Cap Horn fut extrêmement difficile et le navire El Peruano arriva le 4 août 1784 à Rio de Janeiro, où il dut faire une longue pause pour réparation.

25. E.-T. Hamy, op. cit. note 8, pp. XXIII-XXIV.

26. Idem, Ibidem, p 174-176 (Lettres à André Thouin).

27. Id., Ibid., p. LXXXIII.

28. Id., Ibid., p. LXXXV.

29. E.-T. Hamy, op. cit. note 4, p. 95.

30. Irène Aghion, «Le Cabinet des médailles et antiques ", Histoires d'archéologie. De l'objet à l'étude, Paris, Institut national d'histoire de l'art, coll. «Les catalogues d'exposition de l'INHA », 2009, p. 7 [En ligne], mis en ligne le 28 juin 2011 [11/08/2016], http://inha.revues.org/2774.

31. J.-J. Barthélemy, op. cit. note 16.

32. E.-T. Hamy, Les origines du musée d'ethnographie: histoire et documents, Paris, E. Leroux, 1890, p. 26.

33. Bertrand Daugeron, «Entre l'antique et l'exotique, le projet comparatiste oublié du "Muséum des Antiques" en l'an III ", Annales historiques de la Révolution française, 356, avril-juin 2009, p. 148.

34. Idem, Ibidem, p. 147.

35. Id., Ibid., p. 147.

36. Cette exposition a été organisée par Henri Lehmann et présentait 300 objets des plus importantes civilisations de Mésoamérique et des Andes.

37. Cat. d'exp., Chefs-d'œuvre de l'Amérique précolombienne, Musée de l'homme, juin-août 1947, XXVIII ${ }^{\mathrm{e}}$ Congrès international des américanistes, Paris, Société des amis du musée de l'homme, 1947, p. 9.

38. Pascal Riviale, "L'œuvre archéologique d'Alcide d'Orbigny", dans Philippe de Laborde Pédelahore (dir.), Alcide d'Orbigny : à la découverte des nouvelles républiques sud-américaines, Biarritz, Atlantica, 2000, p. 363.

39. Alcide d'Orbigny, Voyage dans l'Amérique méridionale, Paris, P. Bertrand/Strasbourg,Veuve Levrault, 1835 , tome I, p. 5.

40. Idem, Ibidem, p. 5.

41. P. Riviale, op. cit. note 38, p. 366. 
42. Archives du musée de Sèvres, dossier Instructions voyageurs, décembre 1835.

43. Archives du musée de Sèvres, dossier Alcide d'Orbigny, Lettre d'Alexandre Brongniart à Montalivet, le 28 mars 1834.

44. A. d'Orbigny, op. cit. note 39, p. 323.

45. Archives du musée de Sèvres, dossier Alcide d'Orbigny, Lettre de Brongniart à De Wailly, le 10 avril 1834.

46. Riviale nous apprend que la partie de la collection non acquise par Brongniart sera conservée par d'Orbigny jusqu'à sa mort pour être ensuite achetée par un collectionneur, le vicomte de Liesville. Au décès de celui-ci, la collection est censée avoir été donnée au musée de Sèvres et au musée d'Alençon, ville natale de Liesville. Cependant, il n'y a aujourd'hui aucune trace du passage de la collection de d'Orbigny dans ces musées. P. Riviale, op. cit. note 38, p. 383.

47. Archives du musée de Sèvres, dossier Alcide d'Orbigny, Lettre de Brongniart à Montalivet, le 28 mars 1834.

48. Archives du musée de Sèvres, dossier Alcide d'Orbigny, Liste de vases antiques de l'Amérique Méridionale que Brongniart a mis à part, mars 1834.

49. Alexandre Brongniart et Denis-Désiré Riocreux, Description méthodique du musée céramique de la Manufacture royale de porcelaine de Sèvres, Paris, A. Leleux, 1845.

50. Idem, Ibidem, p. 77.

51. Id., Ibid., tome Planches, pl. XVIII.

52. François Reyniers, Sèvres. Musée national de Céramique. Céramiques américaines, Paris, Ed. des Musées nationaux, 1956, p. 110.

53. Archives du musée de Sèvres, dossier Alcide d'Orbigny, Lettre de Brongniart à Montalivet, le 28 mars 1834.

54. Archives nationales, F/17/2970, dossier Auguste Génin, Lettre de Génin au ministre de l'Instruction publique, le 10 janvier 1892

55. Archives nationales, F/17/2970, dossier Auguste Génin, Arrêté du 14 janvier 1892 du ministère de l'Instruction publique.

56. Archives nationales, F/17/2970, dossier Auguste Génin, Lettre de Génin au ministre de l'Instruction publique, le 12 juillet 1892 .

57. Archives nationales, F/17/2970, dossier Auguste Génin, Lettre de Génin au ministre de l'Instruction publique, le 4 mars 1894.

58. Archives nationales, F/17/2970, dossier Auguste Génin, Lettre de Génin au ministre de l'Instruction publique, le 4 mars 1894.

59. Auguste Génin, « Notes d'archéologie mexicaine », Journal de la Société des américanistes, vol. 3, $\mathrm{n}^{\circ} 1,1901$, p. 35.

60. Idem, Ibidem, p. 35.

61. Id., Ibid., p. 37.

62. Génin mentionne que ces pièces correspondent aux $\mathrm{n}^{\text {os }} 140,141,142,143$ et 144 de son catalogue. Claudia De Sevilla identifie ces objets : une figurine, un masque en pierre ainsi qu'un vase tripode, un sifflet et une assiette en terre cuite, correspondant aux objets du musée du quai Branly - Jacques Chirac (MQB) 71.1897.53.254, 71.1897.53.162, 71.1897.53.255, 71.1897.53.201 et 71.1897.53.144. Claudia De Sevilla, Les collections archéologiques d'Auguste Génin : histoire, contexte et méthodes de collecte, musée du quai Branly - Jacques Chirac, note de recherche, 2014, pp. 33-34.

63. Archives nationales, F/17/2970, dossier Auguste Génin, Lettre de Génin au chef de bureau de missions et voyages du ministère de l'Instruction publique, sans date.

64. "J'ai la certitude absolue que le Gouvernement mexicain ne rendra pas les caisses de M. Charnay tant que plusieurs savants comme M. Chavero, par exemple, et la Direction actuelle du Musée national de Mexico, seront là pour s'y opposer. » Archives nationales, F/17/2970, dossier Auguste Génin, Lettre de Génin au ministre de l'Instruction publique, le 4 mars 1894. 
65. Archives nationales, F/17/2970, dossier Auguste Génin, Lettre de Génin au ministre de l'Instruction publique, le 18 mai 1893.

66. Archives nationales, F/17/2970, dossier Auguste Génin, Lettre de Génin au ministre de l'Instruction publique, le 12 juillet 1892.

67. Archives nationales, F/17/2970, dossier Auguste Génin, Lettre de Génin au chef de bureau de missions et voyages du ministère de l'Instruction publique, le 5 juillet 1892 .

68. Archives nationales, F/17/2970, dossier Auguste Génin, Lettre de Génin au ministre de l'Instruction publique, le 29 mai 1894.

69. Auguste Génin, Paul Minnaert, Henri Lavachery, La collection d'antiquités mexicaines de M. Aug. Génin, Bruxelles, Société des américanistes de Belgique, 1931.

70. Cat. d'exp., Les arts anciens de l'Amérique, sous la directon d'Alfred Métraux et Georges Henri Rivière, Paris, Musée des arts décoratifs, Pavillon de Marsan, mai-juin 1928, Paris, G. Van Oest, 1928, p. VII.

71. Cat. d'exp., Chefs-d'œuvre de l'Amérique précolombienne, op. cit. note 37, p. 16.

72. Archives museo de América, Madrid, $\mathrm{n}^{\circ}$ Exp.17/C153, Schmidt-Pizarro. Arrêté d'achat.

73. Ibidem. «S.M. el rey (q.D.g.) ha tenido a bien disponer: $1^{\circ}$ que la lista de objetos incaicos ofrecidos por el Sr. Schmidt y Pizarro sean adquiridos con destino al Museo Arqueológico Nacional. »

74. Archives MQB, 2AM1 A1, DA 000011/37388, Courrier au départ $2^{\mathrm{e}}$ quinzaine de juillet 1930.

75. Archives MQB, 2AM1 A1, DA 000011/37388, Courrier au départ $2^{\mathrm{e}}$ quinzaine de juillet 1930.

76. Pascal Riviale, «Les antiquités péruviennes et la curiosité américaine dans les collections françaises sous l'Ancien Régime », Histoire de l'Art, n² 21/22, mai 1993, p. 43.

77. Par exemple, les missions de Charles Wiener au Pérou et en Bolivie à la fin du XIXe siècle, la mission de Claude Lévi-Strauss au Brésil, Jacques Soustelle au Mexique, entre autres, au début du $\mathrm{xX}^{\mathrm{e}}$ siècle.

78. Pendant cette mission ont été rédigées les «Instructions sommaires pour les collecteurs d'objets ethnographiques ", grâce auxquelles les objets récoltés deviennent de véritables objets « témoins » des cultures étudiées.

79. Pascal Riviale, «L'américanisme français à la veille de la fondation de la Société des américanistes ", Journal de la Société des américanistes, vol. 81, nº 1, 1995, p. 210.

80. I. Aghion, op. cit. note 30, p. 7.

81. B. Daugeron, op. cit. note 33, p. 149.

82. Idem, Ibidem, p. 149.

83. Id., Ibid., p. 150.

84. Id., Ibid., p. 143.

85. Id., Ibid., p. 148.

86. P. Riviale, op. cit. note 79, p. 209.

87. Notice sur le muséum ethnographique des missions scientifiques, rédigée par chacun des missionnaires scientifiques sur les objets qu'il a rapportés, Paris, Palais de l'industrie, 1878, p. 5.

88. Philippe Sabot, "Primitivisme et surréalisme : une "synthèse" impossible? ", Methodos [En ligne], 3, 2003, mis en ligne le 05 avril 2004 [10/10/2016], http://methodos.revues.org/109

89. Paul Rivet et Georges-Henri Rivière, La réorganisation du musée d'ethnographie du Trocadéro, Paris, Musée d'ethnographie du Trocadéro, coll. «Bulletin du musée d'ethnographie du Trocadéro ", 1931, p. 10.

90. Cécilia Hurley-Griener , "Putting sculpture on show/Exposer la sculpture : conclusion », Les Cahiers de l'École du Louvre [En ligne], 8, 2016, p. 59, mis en ligne le $1^{\mathrm{er}}$ mai 2016 [16/10/2016], http://cel.revues.org/344

91. Dominique Jarrassé, « Leçon d'introduction au séminaire doctoral d'histoire de l'art appliquée aux collections 2012-2013 : la qualification de l'objet ", Les Cahiers de l'École du Louvre [En ligne], 4, 2014, p. 2, mis en ligne le $1^{\mathrm{er}}$ avril 2014 [25/04/2016], http://journals.openedition.org/cel/524 


\section{RÉSUMÉS}

Les objets archéologiques et ethnographiques provenant d'Amérique latine que nous trouvons aujourd'hui dans les salles des musées ont eu plusieurs « vies ». Une "vie » qui a commencé au moment de leur fabrication et qui a continué grâce à leur découverte. Etudier leurs histoires, les différents parcours qu'ils ont suivis jusqu'à leur arrivée en Europe et plus particulièrement leur entrée dans les institutions muséales françaises, est une étape importante de l'analyse de la formation des collections en France. Cet article présente le parcours de cinq objets archéologiques, provenant du Pérou et du Mexique, qui font actuellement partie des collections publiques des musées de France.

The archaeological and ethnographic objects from Latin America now in museums have had several "lives". "Lives" that began when they were made and that continued thanks to their discovery. Studying their histories, the different itineraries they have followed until they arrived in Europe and in particular their entries into French museums, is an important step in the analysis of the building up of collections in France. This article presents the itineraries of five archaeological objects, from Peru and Mexico, which are now in the public collections of French museums.

\section{INDEX}

Mots-clés : collectionnisme, objets ethnographiques, objets archéologiques, Amérique, missions scientifiques, Joseph Dombey, Alcide d'Orbigny, Auguste Génin, Guillermo Schmidt-Pizarro

Keywords : collecting, ethnographic objects, archaeological objects, America, scientific missions, Joseph Dombey, Alcide d'Orbigny, Auguste Génin, Guillermo Schmidt-Pizarro

\section{AUTEUR}

\section{VANESSA BERNAL MULTON}

Originaire de Lima, Pérou, Vanessa Bernal Multon a effectué des études d'archéologie andine à l'Université catholique pontificale, en se spécialisant dans les cultures du nord du Pérou comme la culture Mochica et la culture Cajamarca. Après avoir mené plusieurs projets de fouilles et de recherches archéologiques et avoir participé à plusieurs missions de conservation et de mise en valeur des collections archéologiques dans différents musées à Lima, elle est venue en France pour suivre des études d'histoire de l'art à Lyon (licence à l'Université Lyon 2) puis de muséologie à Paris (master à l'École du Louvre). Elle poursuit actuellement des recherches de thèse à l'École $\mathrm{du}$ Louvre sur l'histoire des collections archéologiques et ethnographiques provenant d'Amérique latine présentes dans les principaux musées parisiens, sous la direction de Cecilia Hurley-Griener.

$* * *$

From Lima, Peru, Vanessa Bernal Multon studied Andean archaeology at the Pontificia Universidad Católica del Perú, specialising in the cultures of northern Peru, such as the Mochica and Cajamarca cultures. After having overseen several excavations and archaeological research projects and participated in several conservation missions of archaeological collections in various museums in Lima, she came to France to study art history in Lyon (BA, Université Lyon 
2), then museology in Paris (master's degree, École du Louvre). She is currently carrying out doctoral research at the École du Louvre on the history of archaeological and ethnographic collections from Latin America now in the main Paris museums, under the supervision of Cecilia Hurley-Griener. 